\title{
Nucleosynthesis in Multi-Dimensional Simulations of SNII
}

C. Travaglio ${ }^{1,2}$, K. Kifonidis ${ }^{1}$ and E. Müller ${ }^{1}$

${ }^{1}$ Max-Planck Institut für Astrophysik, Karl-Scwarzschild Str. 1, 85741 Garching bei München, Germany

2 INAF-Osservatorio Astronomico di Pino Torinese, Strada Osservatorio 20, 10025 Torino, Italy;

travaglio@to. astro.it

Summary. We investigate explosive nuclear burning in core collapse supernovae by coupling a tracer particle method to one- and two-dimensional Eulerian hydrodynamic calculations. Adopting the most recent experimental and theoretical nuclear data, we compute the nucleosynthetic yields for $15 M_{\odot}$ stars with solar metallicity, by post-processing the temperature and density history of advected tracer particles. We compare our results to $1 \mathrm{D}$ calculations published in the literature.

\section{Introduction}

The pre- and post-explosive nucleosynthesis of massive stars has been studied extensively by several groups over the last years (see $[6,10,12,13]$, and the references therein). Although a lot of work has been performed in this field, computed nucleosynthetic yields are still affected by numerous uncertainties. The impact of multidimensional hydrodynamics has not been investigated in detail so far. In addition, among the isotopes whose yields are known to depend sensitively on the explosion mechanism, and thus cannot be predicted accurately at present, are key nuclei, like ${ }^{56} \mathrm{Ni}$ and ${ }^{44} \mathrm{Ti}$, that are of crucial importance for the evolution of supernova remnants and for the chemical evolution of galaxies.

\section{Hydrodynamic Models and Marker Particle Method}

The nucleosynthesis calculations presented in this work are based on one and two-dimensional hydrodynamic models of $\mathrm{SNe}$ which follow the revival of the stalling shock, which forms after iron core collapse, and its propagation through the star from $20 \mathrm{~ms}$ up to a few seconds after core bounce (when the explosion energy has saturated and all important nuclear reactions have frozen out). The simulations are started from post-collapse models of Rampp \& Janka (private communication), who followed core-collapse and bounce in the $15 M_{\odot}, Z=Z_{\odot}$ progenitors of [13] and [6]. We employ the HERAKLES code, which solves the hydrodynamic equations in 1, 2 or 3 spatial dimensions 


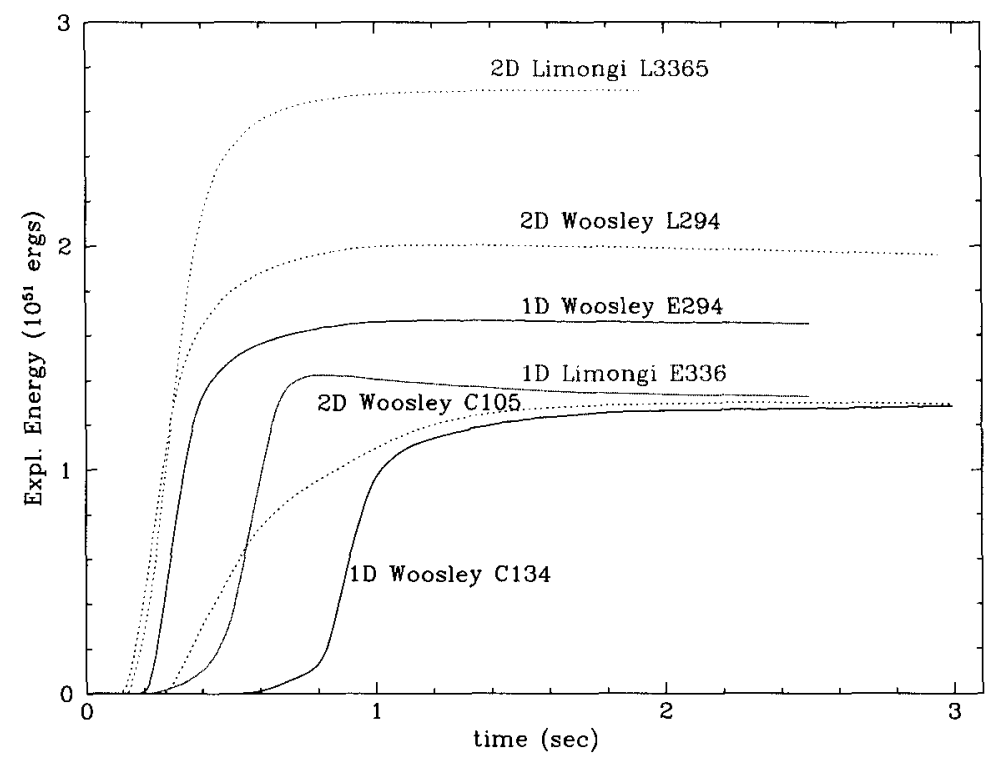

Fig. 1. Explosion energies for the different models discussed in the text.

with the direct Eulerian version of the Piecewise Parabolic Method [2], and which incorporates the light-bulb neutrino treatment and the equation of state of [3] (for more details see [5], and the references therein). The main advantages of our approach are that we drive the shock by accounting for neutrino-matter interactions in the layers outside the newly born neutron star, instead of using a piston (see, e.g., [13]) or a "thermal bomb," and the possibility to perform calculations from one up to three spatial dimensions.

Choosing a hydrodynamic scheme for computing multi-dimensional hydrodynamic models that include the nucleosynthesis, one faces the dilemma of using either a Lagrangian or an Eulerian method. Since nuclear networks with hundreds of isotopes are prohibitively expensive in terms of CPU time and memory for multi-dimensional calculations, such networks can only be solved in a post-processing step (the energy source term due to nuclear burning can usually be calculated with a small network online with the hydrodynamics, and may even be neglected completely in some cases, depending on the structure of the progenitor). Using an Eulerian scheme (where the grid is fixed in space) or even adaptive schemes (in which the grid automatically adapts to resolve steep gradients in the solution) the problem arises how one should obtain the necessary data for the post-processing calculations. We do this by adding a "Lagrangian component" to our Eulerian scheme in the form of marker particles that we passively advect with the flow in the course of the Eulerian calculation, recording their $T$ and $\rho$ history by interpolating the corresponding quantities from the underlying Eulerian grid. A similar method has been adopted in a previous study of multi-dimensional nucleosynthesis 
Table 1. Parameters of models, using the [13](WW95) and [6](LSC00) progenitors.

\begin{tabular}{c|ccccc|c}
\hline \hline Model & Zones & $N_{\text {markers }}$ & $L_{\nu_{e}, 52}^{0}$ & $E_{\text {exp,51 }}$ & $t_{\text {exp }}(\mathrm{ms})$ & ${ }^{56} \mathrm{Ni}\left(\mathrm{M}_{\odot}\right)$ \\
\hline E294 & 2000 & 1024 & 2.940 & 1.46 & 230 & 0.192 \\
L294 & $400 \times 180$ & 8000 & 2.940 & 1.99 & 125 & 0.120 \\
E336 & 2000 & 1024 & 3.365 & 1.33 & 260 & 0.234 \\
L3365 & $400 \times 180$ & 8000 & 3.365 & 2.69 & 150 & 0.146 \\
C134 & 2000 & 1024 & 1.344 & 1.28 & 600 & 0.085 \\
C105 & $400 \times 180$ & 8000 & 1.050 & 1.29 & 280 & 0.064 \\
\hline \hline
\end{tabular}

in core collapse SNe by [8], in very massive stars [7], and in Type Ia SNe [9]. For our 1D and 2D calculations we have used 1024 and 8000 marker particles, respectively. They are distributed homogeneously in mass throughout the progenitor's Fe core, $\mathrm{Si}, \mathrm{O}$, and $\mathrm{C}$ shells assuming the composition of the progenitor at the corresponding mass coordinate as the initial composition of the respective tracer particle.

\section{Nucleosynthesis: Results and Perspectives}

Given the temperature and density history of individual marker particles we can calculate their nucleosynthetic evolution and compute the total yields (including the decays of unstable isotopes) as a sum over all particles. The reaction network employed for our nucleosynthesis calculations contains 296 nuclear species, from neutrons, protons, and $\alpha$-particles to ${ }^{78} \mathrm{Ge}$ (F.-K. Thielemann, private communication). The reaction rates include experimental and theoretical nuclear data as well as weak interaction rates.

So far we have investigated six explosion models for their nucleosynthetic yields: a one-dimensional (E294) and a corresponding two-dimensional (L294) model that made use of model s15s7b of [13], with high energy of the explosion. A second pair of a one (E336) and two-dimensional (E3365) simulation for the $15 M_{\odot}[6]$ progenitor. Also in this second case the explosion energy obtained is high. Finally, a third pair of a one (C134) and two-dimensional (C105) simulation for the $15 M_{\odot}$ s15s7b of [13], but with a much lower explosion energy. The properties of these models are given in Table 1, where $L_{\nu_{e}, 52}^{0}$ is the electron neutrino luminosity (in units of $10^{52} \mathrm{erg} / \mathrm{s}$ ), $E_{\text {exp }, 51}$ is the explosion energy (in units of $10^{51} \mathrm{erg}$ ), and $t_{\exp }$ is the explosion time scale (in $\mathrm{ms}$ ) defined as the time after the start of the simulation when the explosion energy exceeds $10^{49} \mathrm{erg}$ (for a detailed explanation of the neutrino parameters see $[3,5]$ ). In the last column of Table 1 we also added the ${ }^{56} \mathrm{Ni}$ mass obtained using these hydrodynamic models and the nucleosynthesis calculations described above.

Figure 1 shows the evolution of the explosion energy for the six models, using the same neutrino luminosity for the $1 \mathrm{D}$ and $2 \mathrm{D}$ model of the same 


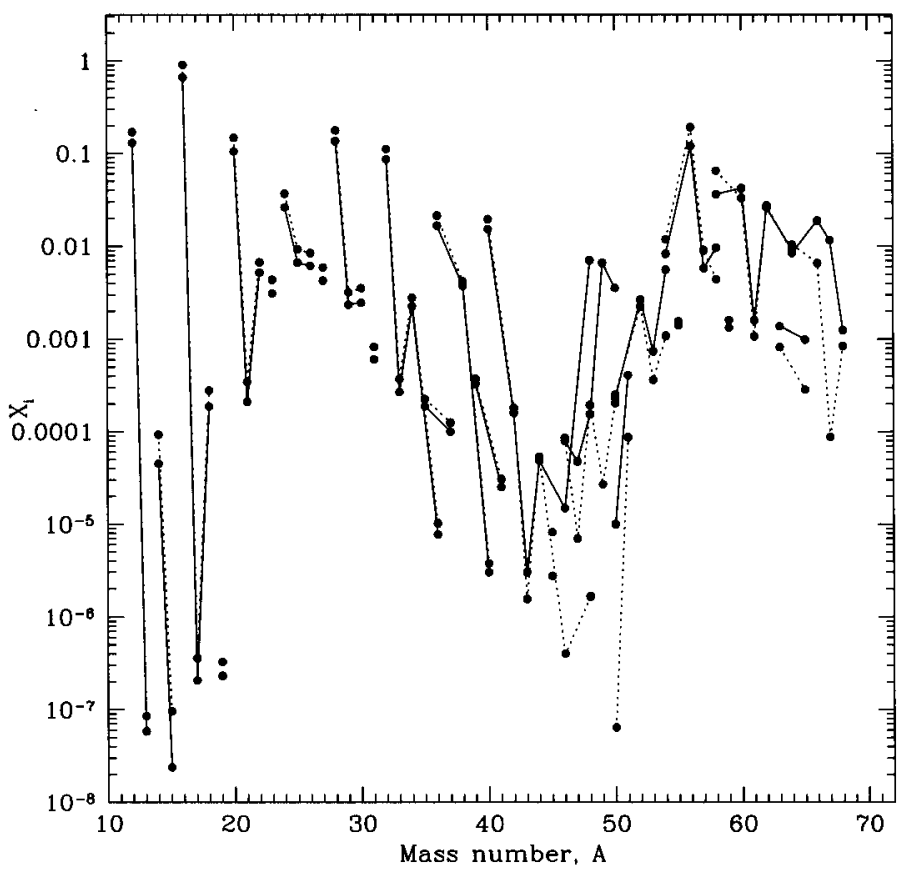

Fig. 2. Final mass fractions obtained for the $1 \mathrm{D}$ (dotted line) and $2 \mathrm{D}$ simulation (solid line) as a function of the atomic number, for the cases E294 and L294.

progenitor. The [6] progenitor needs higher neutrino luminosity to explode, mainly due to the fact that it has a more compact core. The C134 and C105 models evolve much slower in time (as an effect of a lower explosion energy). Our goal is to investigate the consequences on nucleosynthesis (in particular mixing can play a major role under these conditions).

In Fig. 2 we compare the yields of the 1D and 2D simulations (E294 and L294, respectively) for the [13] progenitor. The differences, which are apparently negligible in case of the lighter nuclei and small for the heavier ones, are mainly due to the on average higher temperatures in the $2 \mathrm{D}$ simulation, i.e. more free neutrons are available in the innermost layers of the $2 \mathrm{D}$ simulation. This results in higher production factors for isotopes very sensitive to neutron captures, like e.g. ${ }^{46,48} \mathrm{Ca},{ }^{49,50} \mathrm{Ti},{ }^{50,51} \mathrm{~V},{ }^{54} \mathrm{Cr}$, and ${ }^{67} \mathrm{Zn}$. A more detailed discussion on nucleosynthesis calculation in multidimensional simulations of SNII is included in Travaglio et al. (2004, in preparation), where also a network extended to heavier isotopes has been considered.

For the cases with high energy of the explosion, the reason for the rather small differences in the yields between the $1 \mathrm{D}$ and $2 \mathrm{D}$ simulations are the high initial neutrino luminosities, that we adopted for our calculations, and their rapid exponential decline. This leads to very rapid (and energetic) explosions. The short explosion time scale prevents the convective bubbles, 
which form due to the negative entropy gradient in the neutrino-heated region, to merge to large-scale structures that can lead to global anisotropies, and hence to significant differences compared to the $1 \mathrm{D}$ case. Lowering the neutrino luminosities (and the explosion energies), as in the cases C134 and C105, we obtain stronger convection that strongly distorts the shock wave by developing large bubbles of neutrino-heated material (see [3, 4] for examples). Adopting constant core luminosities instead of an exponential law, we can produce models where the phase of convective overturn lasts for several turn-over times and which exhibit the vigorous boiling behavior reported by [1]. Such cases can finally develop global anisotropies, showing a dominance of the $m=0, l=1$ mode of convection (see [11]). As a consequence, convection can lead to large deviations from spherical symmetry, and thus to larger differences in the final yields than those visible in Fig. 2. We are currently investigating such models in more detail.

\section{References}

1. A. Burrows, J. Hayes, B.A. Fryxell: Astrophys. J. 450, 830 (1995)

2. P. Colella, P.R. Woodward: J. Comput. Phys. 54, 174 (1984)

3. H.-Th. Janka, E. Müller: Astron. Astrophys. 306, 167 (1996)

4. K. Kifonidis, T. Plewa, H.-Th. Janka, E. Müller: Astrophys. J. Lett. 531, L123 (2000)

5. K. Kifonidis, T. Plewa, H.-Th. Janka, E. Müller: Astron. Astrophys. 408, $621(2003)$

6. M. Limongi, O. Straniero, S. Chieffi: Astrophys. J. Suppl. 129, 625 (2000)

7. K. Maeda, T. Nakamura, K. Nomoto, P. Mazzali, F. Patat, I. Hachisu: Astrophys. J. 565, 405 (1992)

8. S. Nagataki, M.-A. Hashimoto, K. Sato, S. Yamada: Astrophys. J. 486, 1026 (1997)

9. J. Niemeyer, M. Reinecke, C. Travaglio, W. Hillebrandt 2002: In Workshop From Twilight to Highlight: The Physics of Supernovae, p. 151

10. T. Rauscher, A. Heger, R.D. Hoffman, S.E. Woosley: Astrophys. J. 576, $323(2002)$

11. L. Scheck, T. Plewa, H.-Th. Janka, K. Kifonidis, E. Müller: astro-ph 0307352

12. F.-K. Thielemann, K. Nomoto, M.-A. Hashimoto: Astrophys. J. 460, 408 (1996)

13. S.E. Woosley, T.A. Weaver: Astrophys. J. Suppl. 101, 181 (1995) 